Homology, Homotopy and Applications, vol.14(1), 2012, pp.19-32

\title{
A HOMOTOPY COLIMIT THEOREM FOR DIAGRAMS OF BRAIDED MONOIDAL CATEGORIES
}

\author{
A.R. GARZÓN AND R. PÉREZ \\ (communicated by Graham Ellis)
}

\begin{abstract}
Thomason's Homotopy Colimit Theorem has been extended to bicategories and this extension can be adapted, through the delooping principle, to a corresponding theorem for diagrams of monoidal categories. In this version, we show that the homotopy type of the diagram can also be represented by a genuine simplicial set nerve associated with it. This suggests the study of a homotopy colimit theorem, for diagrams $\mathcal{B}$ of braided monoidal categories, by means of a simplicial set nerve of the diagram. We prove that it is weak homotopy equivalent to the homotopy colimit of the diagram, of simplicial sets, obtained from composing $\mathcal{B}$ with the geometric nerve functor of braided monoidal categories.
\end{abstract}

\section{Introduction}

The Grothendieck construction $\int_{I} \mathcal{C}[\mathbf{1 3}]$ on a diagram of categories $\mathcal{C}: I^{\text {op }} \rightarrow$ Cat has recently been extended to the context of 2-categories [10] and, more broadly, of bicategories [5]. These results allowed Thomason's well-known Homotopy Colimit Theorem [19] to be extended to diagrams of 2-categories [10, Theorem 4.5] and diagrams of bicategories [5, Theorem 7.3]. Through the elemental delooping construction $[\mathbf{1 5}]$, a monoidal category $\mathcal{M}=(\mathcal{M}, \otimes, a, \mathrm{I}, l, r)[\mathbf{1 6}]$ can be regarded as a bicategory $\Omega^{-1} \mathcal{M}$ with only one object, and therefore the latter extension provides a corresponding theorem for diagrams of monoidal categories. In this theorem, we note that the homotopy type of the homotopy colimit of the diagram can also be represented by a genuine simplicial set nerve associated with the diagram. A braided monoidal category $\mathcal{M}=(\mathcal{M}, \otimes, a, \mathrm{I}, l, r, \boldsymbol{c})[\mathbf{1 4}]$ defines, by double delooping, a oneobject, one-arrow tricategory $\Omega^{-2} \mathcal{M}[\mathbf{3}, \mathbf{1 5}]$. Although neither the Grothendieck construction nor the homotopy colimit theorem is known for diagrams of tricategories, the above remark suggests an extension of Thomason's theorem to diagrams of braided monoidal categories $\mathcal{B}: I^{\mathrm{op}} \rightarrow$ BrMonCat. The composite of $\mathcal{B}$ with the geometric nerve Ner of braided monoidal categories $[\mathbf{6}]$ gives a diagram of simplicial sets whose

This work has been partially supported by DGI of Spain and FEDER (Project: MTM2007-65431); Consejería de Innovación de J. de Andalucía (FQM-168).

Received March 21, 2011, revised July 24, 2011; published on April 26, 2012. 2000 Mathematics Subject Classification: 18D05, 18D10, 55P15, 55P48.

Key words and phrases: homotopy colimit, simplicial set, bicategory, braided monoidal category. Article available at http://intlpress.com/HHA/v14/n1/a2 and doi:10.4310/HHA.2012.v14.n1.a2 Copyright (C) 2012, International Press. Permission to copy for private use granted. 
homotopy colimit is the focus of our study. We use a notion of nerve $\operatorname{Ner}_{I} \mathcal{B}$, associated with the diagram $\mathcal{B}$, which is a particular case of a general notion for the nerve of a pseudofunctor of braided monoidal categories introduced in [7]. In fact, we show that $\operatorname{Ner}_{I} \mathcal{B}$ can represent the homotopy type of the diagram through the existence (Theorem 3.1) of a natural weak homotopy equivalence $\eta$ : hocolim $\operatorname{Ner} \mathcal{B} \rightarrow \operatorname{Ner}_{I} \mathcal{B}$.

\section{Preliminaries}

We start by fixing notations and terminology and reviewing necessary results from the background of (bi)simplicial sets used throughout the paper. We employ the standard symbolism and nomenclature to be found in texts on simplicial homotopy theory (see $[\mathbf{1 1}, \mathbf{1 7}]$ ).

Hereafter, we shall regard each ordered set $[n]=\{0,1, \ldots, n\}$ as the category with exactly one arrow $j \rightarrow i$ if $i \leqslant j$. Then, a non-decreasing map $[m] \rightarrow[n]$ is a functor so that we can see $\Delta$, the simplicial category of finite ordinal numbers, as a full subcategory of Cat, the category of small categories. Simpl.Set denotes the category of simplicial sets, that is, functors $S: \Delta^{\mathrm{op}} \rightarrow$ Set. A weak homotopy equivalence of simplicial sets is a simplicial map whose geometric realization is a homotopy equivalence.

A bisimplicial set is a functor $S: \Delta^{\mathrm{op}} \times \Delta^{\mathrm{op}} \rightarrow$ Set. This amounts to a family of sets $\left\{S_{p, q} ; p, q \geqslant 0\right\}$ together with horizontal and vertical face and degeneracy operators

$$
S_{p+1, q} \stackrel{s_{i}^{h}}{\longleftarrow} S_{p, q} \stackrel{d_{i}^{h}}{\longrightarrow} S_{p-1, q}, \quad S_{p, q+1} \stackrel{s_{j}^{v}}{\longleftarrow} S_{p, q} \stackrel{d_{j}^{v}}{\longrightarrow} S_{p, q-1},
$$

with $0 \leqslant i \leqslant p$ and $0 \leqslant j \leqslant q$ respectively, such that, for all $p$ and $q$, both $S_{p, *}$ and $S_{*, q}$ are simplicial sets and the horizontal operators commute with the vertical ones. Bisimpl.Set denotes the category of bisimplicial sets.

We shall use the bar construction on a bisimplicial set $\bar{W} S$, also called its codiagonal or total complex. Let us recall that the functor

\section{$\bar{W}:$ Bisimpl.Set $\rightarrow$ Simpl.Set}

can be described, for any given bisimplicial set $S$, as follows $[\mathbf{1}, \S I I I]$ : the set of $p$-simplices of $\bar{W} S$ is

$$
\left\{\left(t_{0, p}, \ldots, t_{p, 0}\right) \in \prod_{m=0}^{p} S_{m, p-m} \mid d_{0}^{v} t_{m, p-m}=d_{m+1}^{h} t_{m+1, p-m-1}, 0 \leqslant m<p\right\}
$$

and, for $0 \leqslant i \leqslant p$, the faces and degeneracies of a $p$-simplex are given by

$$
\begin{aligned}
& d_{i}\left(t_{0, p}, \ldots, t_{p, 0}\right)=\left(d_{i}^{v} t_{0, p}, \ldots, d_{i}^{v} t_{i-1, p-i+1}, d_{i}^{h} t_{i+1, p-i-1}, \ldots, d_{i}^{h} t_{p, 0}\right), \\
& s_{i}\left(t_{0, p}, \ldots, t_{p, 0}\right)=\left(s_{i}^{v} t_{0, p}, \ldots, s_{0}^{v} t_{i, p-i}, s_{i}^{h} t_{i, p-i}, \ldots, s_{i}^{h} t_{p, 0}\right) .
\end{aligned}
$$

On the other hand, by composing with the diagonal functor diag: $\Delta^{\mathrm{op}} \rightarrow \Delta^{\mathrm{op}} \times \Delta^{\mathrm{op}}$, the bisimplicial set $S$ also provides another simplicial set $\operatorname{diag} S:[n] \mapsto S_{n, n}$, whose face and degeneracy operators are given in terms of those of $S$ by the formulas $d_{i}=$ $d_{i}^{h} d_{i}^{v}$ and $s_{i}=s_{i}^{h} s_{i}^{v}$, respectively. 
For any bisimplicial set $S$, there is a natural weak homotopy equivalence $[\mathbf{8}, \mathbf{9}]$

$$
\Phi: \operatorname{diag} S \rightarrow \bar{W} S
$$

that carries a $p$-simplex $t_{p, p} \in \operatorname{diag} S$ to

$$
\Phi t_{p, p}=\left(\left(d_{1}^{h}\right)^{p} t_{p, p},\left(d_{2}^{h}\right)^{p-1} d_{0}^{v} t_{p, p}, \ldots,\left(d_{m+1}^{h}\right)^{p-m}\left(d_{0}^{v}\right)^{m} t_{p, p}, \ldots,\left(d_{0}^{v}\right)^{p} t_{p, p}\right) .
$$

The next subsection is devoted to recalling the extension to bicategories, shown in [5], of Thomason's Homotopy Colimit Theorem.

\subsection{Thomason's Homotopy Colimit Theorem for bicategories}

Recall that the Grothendieck nerve functor Ner: Cat $\rightarrow$ Simpl.Set associates, with every small category $\mathcal{C}$, the $\operatorname{simplicial}$ set $\operatorname{Ner} \mathcal{C}$ whose $n$-simplices are all functors $F:[n] \rightarrow \mathcal{C}$ or, equivalently, tuples of arrows in $\mathcal{C}, F=\left(F j \stackrel{F_{i, j}}{\longrightarrow} F i\right)_{0 \leqslant i \leqslant j \leqslant n}$, such that $F_{i, j} F_{j, k}=F_{i, k}$ for $i \leqslant j \leqslant k$ and $F_{i, i}=1_{F i}$. For any given small category $I$ and any diagram of categories $\mathcal{C}: I^{\mathrm{op}} \rightarrow$ Cat, the composite $\operatorname{Ner} \mathcal{C}: I^{\mathrm{op}} \rightarrow$ Simpl.Set is just a diagram of simplicial sets. The homotopy colimit construction by Bousfield and Kan [4] of this diagram is the simplicial set

$$
\begin{aligned}
\operatorname{hocolim}_{I} \operatorname{Ner} \mathcal{C}: \Delta^{\mathrm{op}} & \rightarrow \text { Set } \\
{[n] } & \mapsto \coprod_{[n] \stackrel{\sigma}{\rightarrow} I} \operatorname{Func}\left([n], \mathcal{C}_{\sigma 0}\right) .
\end{aligned}
$$

Considering the Grothendieck construction $\int_{I} \mathcal{C}$ of the diagram $\mathcal{C}: I^{\text {op }} \rightarrow$ Cat, Thomason's Homotopy Colimit Theorem states the following:

Theorem 2.1 ([19, Theorem 1.2]). For any diagram of categories $\mathcal{C}: I^{\mathrm{op}} \rightarrow$ Cat, there is a natural weak homotopy equivalence

$$
\eta: \operatorname{hocolim}_{I} \operatorname{Ner} \mathcal{C} \rightarrow \operatorname{Ner} \int_{I} \mathcal{C}
$$

In this way, the classifying space of the category $\int_{I} \mathcal{C}$ (that is, the geometric realization of its nerve) can be thought of as a homotopy colimit of the classifying spaces of the categories $\mathcal{C}_{i}$ that arise from the initial input data $i \rightarrow \mathcal{C}_{i}$ given by the diagram of categories $\mathcal{C}$.

Below we quickly review the extension to bicategories given in [5] of the above theorem. For background concerning bicategories, we refer the reader to $[\mathbf{2 , ~ 1 2 , ~ 1 8 ] . ~}$ Bicat denotes the category of bicategories and homomorphisms between them (i.e., lax functors where the structure constraints are invertible). For any diagram of bicategories $\mathcal{C}: I^{\text {op }} \rightarrow$ Bicat, there is [5] a bicategorical Grothendieck construction $\int_{I} \mathcal{C}$ that suitably assembles all bicategories $\mathcal{C}_{i}, i \in \mathrm{Ob} I$. Also, for any small bicategory $\mathcal{C}$, we recall that the geometric nerve of $\mathcal{C}$ is the simplicial set

$$
\begin{aligned}
\Delta \mathcal{C}: \Delta^{\text {op }} & \rightarrow \text { Set } \\
{[n] } & \mapsto \operatorname{LaxFunc}([n], \mathcal{C})
\end{aligned}
$$

whose $n$-simplices are all lax functors $F:[n] \rightarrow \mathcal{C}$. The unitary geometric nerve of $\mathcal{C}$, $\Delta^{u} \mathcal{C}$, is defined considering only normal (or unitary, i.e., when the unit constraints are all identities) lax functors $F:[n] \rightarrow \mathcal{C}$. 
Then, Thomason's theorem admits the following generalization to diagrams of bicategories:

Theorem 2.2 ([5, Theorem 7.3]). For any diagram of bicategories $\mathcal{C}: I^{\mathrm{op}} \rightarrow$ Bicat, there exists a natural weak homotopy equivalence of simplicial sets

$$
\eta: \operatorname{hocolim}_{I} \Delta \mathcal{C} \rightarrow \Delta \int_{I} \mathcal{C}
$$

where $\Delta \mathcal{C}: I^{\mathrm{op}} \rightarrow$ Simpl.Set $i$ the diagram of simplicial sets obtained by composing $\mathcal{C}$ with the geometric nerve functor $\Delta:$ Bicat $\rightarrow$ Simpl.Set.

Ten different (but homotopically equivalent) ways of defining the classifying space of any bicategory have been shown in [5, Theorem 6.1]. The above theorem therefore establishes how the classifying space of the bicategorical Grothendieck construction $\int_{I} \mathcal{C}$ can be thought of as a homotopy colimit of the classifying spaces of the bicategories $\mathcal{C}_{i}$ that arise from the initial input data $i \mapsto \mathcal{C}_{i}$ given by the diagram of bicategories $\mathcal{C}$.

In the next subsection, we show how Theorem 2.2 provides a corresponding theorem for monoidal categories in which, in addition, the homotopy colimit can be represented by a genuine simplicial set nerve of the diagram.

\subsection{Homotopy colimit theorem for monoidal categories}

Below, MonCat denotes the category of monoidal categories and monoidal functors between them. Note that every monoidal category $\mathcal{M}=(\mathcal{M}, \otimes, a, \mathrm{I}, l, r)$ can be regarded as a bicategory $\Omega^{-1} \mathcal{M}$ with only one object, $*$, whose morphisms are the objects of $\mathcal{M}$ and whose deformations are the morphisms of $\mathcal{M}$. The horizontal composition is given by the tensor functor $\otimes: \mathcal{M} \times \mathcal{M} \rightarrow \mathcal{M}$, the identity at the object * is the unit object I of $\mathcal{M}$, and the associativity, left and right unit constraints for $\Omega^{-1} \mathcal{M}$ are just those of the monoidal category. This observation, due to J. Benabou [2], that monoidal categories are essentially the same as bicategories with just one object, is known as the delooping principle, and the bicategory $\Omega^{-1} \mathcal{M}$ is called the delooping of the monoidal category $[\mathbf{1 5}, 2.10]$. Thus, we have the delooping embedding $\Omega^{-1}$ : MonCat $\rightarrow$ Bicat.

If $\mathcal{M}: I^{\text {op }} \rightarrow$ MonCat, $(j \stackrel{a}{\rightarrow} i) \mapsto\left(\mathcal{M}_{i} \stackrel{a^{*}}{\rightarrow} \mathcal{M}_{j}\right)$, is a diagram of monoidal categories, then it follows from Theorem 2.2 that the homotopy type of $\mathcal{M}$ is modelled by the bicategory $\int_{I} \Omega^{-1} \mathcal{M}$, after the existence, according to (2), of a weak homotopy equivalence

$$
\eta: \operatorname{hocolim}_{I} \Delta \Omega^{1} \mathcal{M} \rightarrow \Delta \int_{I} \Omega^{-1} \mathcal{M}
$$

But, as we shall see in detail below, the homotopy type of the diagram $\mathcal{M}$ can also be represented by a simplicial set associated to it, namely $\operatorname{Ner}_{I} \mathcal{M}$, the nerve of the diagram defined in [7].

Recall that a 2-cocycle of $I$ with coefficients in $\mathcal{M}$ is a system of data $(Y, f)$ consisting of:

- For each arrow $j \stackrel{a}{\rightarrow} i$ in $I$, an object $Y_{a} \in \mathcal{M}_{j}$. 
- For each pair of composable arrows in $I, k \stackrel{b}{\rightarrow} j \stackrel{a}{\rightarrow} i$, a morphism in $\mathcal{M}_{k}$

$$
b^{*} Y_{a} \otimes Y_{b} \stackrel{f_{a, b}}{\longrightarrow} Y_{a b},
$$

such that $Y_{1_{j}}=\mathrm{I}$ (the unit object of $\mathcal{M}_{j}$ ), the morphisms $f_{1, a}: a^{*} \mathrm{I} \otimes Y_{a} \rightarrow Y_{a}$ and $f_{a, 1}: Y_{a} \otimes \mathrm{I} \rightarrow Y_{a}$ are the canonical isomorphisms given by the unit constraints of the monoidal category $\mathcal{M}_{j}$ and the monoidal functor $a^{*}$, and for any three composable triplet, $\ell \stackrel{c}{\rightarrow} k \stackrel{b}{\rightarrow} j \stackrel{a}{\rightarrow} i$, of morphisms in $I$, the coherence condition given by the commutativity of the following diagram in $\mathcal{M}_{\ell}$ :

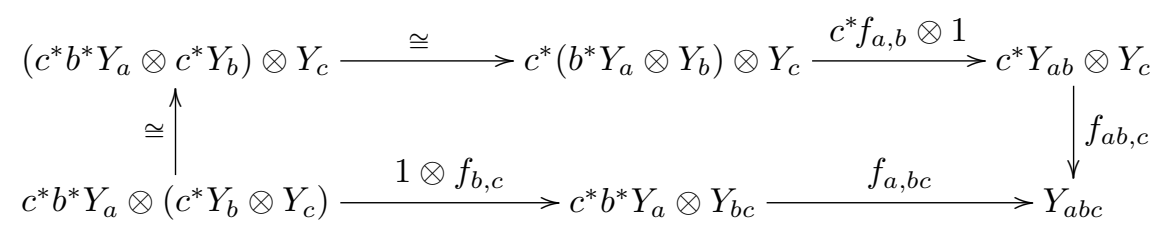

(where the unnamed isomorphisms are canonical) must hold.

Then, $\operatorname{Ner}_{I} \mathcal{M}$, the nerve of the diagram, is defined as the simplicial set

$$
\operatorname{Ner}_{I} \mathcal{M}:[n] \mapsto \bigsqcup_{G:[n] \rightarrow I} Z^{2}([n], \mathcal{M} G),
$$

where $G:[n] \rightarrow I$ is any functor and $Z^{2}([n], \mathcal{M} G)$ is the set of 2-cocycles of $[n]$ in the composite functor $[n] \stackrel{G}{\longrightarrow} I \stackrel{\mathcal{M}}{\longrightarrow}$ MonCat.

Recalling that the unitary geometric nerve of any small bicategory $\mathcal{C}$ is the simplicial set

$$
\begin{aligned}
\Delta^{u} \mathcal{C}: \Delta^{\text {op }} & \rightarrow \text { Set } \\
{[n] } & \mapsto \operatorname{NorLaxFunc}([n], \mathcal{C})
\end{aligned}
$$

whose $n$-simplices are all normal (or unitary, i.e., when the unit constraints are all identities) lax functors $F:[n] \rightarrow \mathcal{C}$, now we can prove the following:

Proposition 2.3. For any diagram of monoidal categories $\mathcal{M}: I^{\mathrm{op}} \rightarrow$ MonCat, there is a natural isomorphism of simplicial sets

$$
\operatorname{Ner}_{I} \mathcal{M} \cong \Delta^{u} \int_{I} \Omega^{-1} \mathcal{M}
$$

Proof. The isomorphism will be clear after describing the simplices of both simplicial sets in each dimension.

First we observe that, according to the general construction of the bicategorical Grothendieck construction [5], the bicategory $\int_{I} \Omega^{-1} \mathcal{M}$ has, as objects, pairs $\left(*_{i}, i\right)$ where $i \in \mathrm{Ob} I$ and $*_{i}$ is the unique object of $\Omega^{-1} \mathcal{M}_{i}$. Thus, Ob $\int_{I} \Omega^{-1} \mathcal{M} \cong \mathrm{Ob} I$. A morphism from $\left(*_{j}, j\right)$ to $\left(*_{i}, i\right)$ is a pair $(X, a)$, where $a: j \rightarrow i$ is a morphism in $I$ and $X \in \mathrm{Ob} \mathcal{M}_{j}$, and a 2-cell from $(X, a)$ to $(Y, a)$ is just a morphism $\alpha: X \rightarrow Y$ in 
$\mathcal{M}_{j}$. The horizontal composition functor is given by

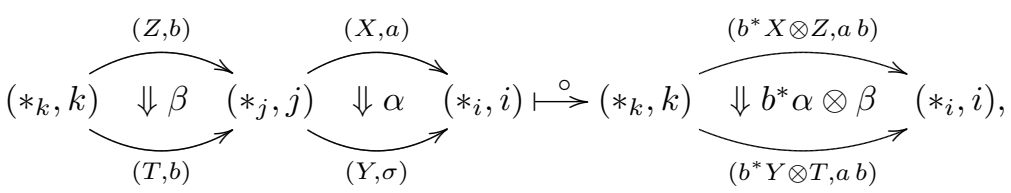

where $b^{*}: \Omega^{-1} \mathcal{M}_{j} \rightarrow \Omega^{-1} \mathcal{M}_{k}$ is the homomorphism induced by $b: k \rightarrow j$.

Then, the unitary geometric nerve of the bicategory $\int_{I} \Omega^{-1} \mathcal{M}$ is the simplicial set whose $n$-simplices, the normal lax functors $[n] \rightarrow \int_{I} \Omega^{-1} \mathcal{M}$, are described as follows (see [5, Lemma 4.2]):

- In dimension zero, $\left(\Delta^{u} \int_{I} \Omega^{-1} \mathcal{M}\right)_{0}=\mathrm{Ob} \int_{I} \Omega^{-1} \mathcal{M} \cong \mathrm{Ob} I$.

- The 1-simplices $F:[1] \rightarrow \int_{I} \Omega^{-1} \mathcal{M}$ of $\Delta^{u} \int_{I} \Omega^{-1} \mathcal{M}$ are the morphisms in $\int_{I} \Omega^{-1} \mathcal{M}$ from $F 1=\left(*_{F 1}, F 1\right)$ to $F 0=\left(*_{F 0}, F 0\right)$, that is, pairs $\left(X_{0,1}, F_{0,1}\right)$, where $X_{0,1}: *_{F 1} \rightarrow *_{F 1}$ is a morphism in $\Omega^{-1} \mathcal{M}_{F 1}$ (i.e., an object of $\left.\mathcal{M}_{F 1}\right)$ and $F_{0,1}: F 1 \rightarrow$ $F 0$ is a morphism in $I$.

- The 2-simplices $F:[2] \rightarrow \int_{I} \Omega^{-1} \mathcal{M}$ are triangles

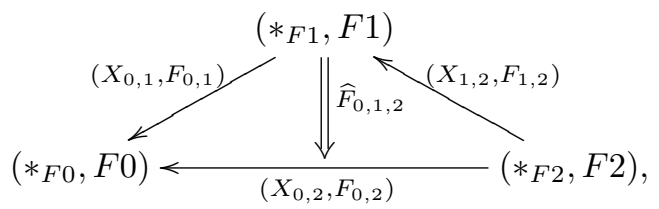

where $\widehat{F}_{0,1,2}:\left(X_{0,1}, F_{0,1}\right) \circ\left(X_{1,2}, F_{1,2}\right) \Rightarrow\left(X_{0,2}, F_{0,2}\right)$ is a deformation in $\int_{I} \Omega^{-1} \mathcal{M}$. Then, $\widehat{F}_{0,1,2}:\left(F_{1,2}^{*} X_{0,1} \otimes X_{1,2}, F_{0,1} F_{1,2}\right) \Rightarrow\left(X_{0,2}, F_{0,2}\right)$, and therefore $F_{0,1} F_{1,2}=F_{0,2}$ and $\widehat{F}_{0,1,2}$ is just a deformation $F_{0,1,2}: F_{1,2}^{*} X_{0,1} \otimes X_{1,2} \rightarrow X_{0,2}$ in $\Omega^{-1} \mathcal{M}_{F 2}$, that is, a morphism in $\mathcal{M}_{F 2}$ from $F_{1,2}^{*} X_{0,1} \otimes X_{1,2}$ to $X_{0,2}$.

Then, a 2-simplex of $\Delta^{u} \int_{I} \Omega^{-1} \mathcal{M}$ is a tuple $\left(F 0, F 1, F 2, F_{0,1}, F_{0,2}, F_{1,2}, F_{0,1,2}\right)$, where $F i, i=0,1,2$ are objects of $I, F_{i, j}: F j \rightarrow F i, 0 \leqslant i<j \leqslant 2$, are morphisms in $I$ such that $F_{0,1} F_{1,2}=F_{0,2}$, and $F_{0,1,2}: F_{1,2}^{*} X_{0,1} \otimes X_{1,2} \rightarrow X_{0,2}$ is a morphism in $\mathcal{M}_{F 2}$ with $X_{0,1} \in \mathrm{Ob} \mathcal{M}_{F 1}$ and $X_{1,2}, X_{0,2} \in \mathrm{Ob} \mathcal{M}_{F 2}$.

- If $n \geqslant 3$, then a $n$-simplex $F:[n] \rightarrow \int_{I} \Omega^{-1} \mathcal{M}$ is determined by objects $F i$ of $I, 0 \leqslant i \leqslant n$; for any $0 \leqslant i<j \leqslant n$, by objects $X_{i, j} \in \mathrm{Ob} \mathcal{M}_{F j}$ and morphisms in $I, F_{i, j}: F j \rightarrow F i$, such that, for any $0 \leqslant i<j<k \leqslant n, F_{i, j} F_{j, k}=F_{i, k}$; and, for any $0 \leqslant i<j<k \leqslant n$, by morphisms in $\mathcal{M}_{F k}, F_{i, j, k}: F_{j, k}^{*} X_{i, j} \otimes X_{j, k} \rightarrow X_{i, k}$ such that, for any $0 \leqslant i<j<k<\ell \leqslant n$, the following diagram in $\mathcal{M}_{F \ell}$, where 'can' denotes a composite of canonical isomorphisms, is commutative:

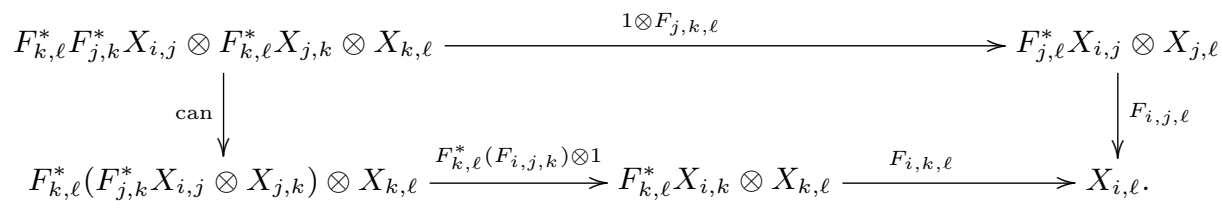

The whole data giving the normal lax functor $F:[n] \rightarrow \int_{I} \Omega^{-1} \mathcal{M}$ are obtained by putting $X_{i, i}=I, 0 \leqslant i \leqslant n$, where $I$ is the unit object of $\mathcal{M}_{F i}$, and $F_{i, i, j}: F_{i, j}^{*} I \otimes$ $X_{i, j} \rightarrow X_{i, j}$ and $F_{i, j, j}: X_{i, j} \otimes I \rightarrow X_{i, j}, 0 \leqslant i<j \leqslant n$, given by canonical (left, right, unit) constraints of the monoidal category $\mathcal{M}_{F j}$. 
As for the $n$-simplices of $\operatorname{Ner}_{I} \mathcal{M}$,

$$
\bigsqcup_{G:[n] \rightarrow I} Z^{2}([n], \mathcal{M} G)
$$

they can be described as follows:

- A 0-simplex consists of a functor $G:[0] \rightarrow I$ and a 2-cocycle of [0] with coefficients in $\mathcal{M} G$. Thus, it is determined by the objects $G 0$ of $I$ and therefore

$$
\left(\operatorname{Ner}_{I} \mathcal{M}\right)_{0} \cong \mathrm{Ob}(I) \cong\left(\Delta^{u} \int_{I} \Omega^{-1} \mathcal{M}\right)_{0}
$$

- A 1-simplex is given by a functor $G:[1] \rightarrow I$ and a 2-cocycle of [1] with coefficients in $\mathcal{M} G$. A functor $G:[1] \rightarrow I$ is just a 1 -simplex of the nerve of the category $I$, that is, a system $\left(G i, G_{i, j}: G j \rightarrow G i\right)$, where $G i, i=0,1$ are objects of $I$ and $G_{i, j}, 0 \leqslant i \leqslant$ $j \leqslant 1$, is a morphism in $I$ with $G_{i, i}=1_{G i}, i=0,1$. A 2-cocycle of [1] with coefficients is $\mathcal{M} G$ consists of objects $Y_{i, j} \in \mathrm{Ob} \mathcal{M}_{G j}, 0 \leqslant i \leqslant j \leqslant 1$, with $Y_{i, i}=\mathrm{I}$ the unit object of $\mathcal{M}_{G i}$. Thus, a 1 -simplex consists of a system of data $\left\{\left(G i, G_{i, j}: G j \rightarrow G i, Y_{i, j}\right)\right\}$, $0 \leqslant i \leqslant j \leqslant 1$, as above and therefore

$$
\left(\operatorname{Ner}_{I} \mathcal{M}\right)_{1} \cong\left(\Delta^{u} \int_{I} \Omega^{-1} \mathcal{M}\right)_{1}
$$

- A 2-simplex is given by a functor $G:[2] \rightarrow I$ and a 2-cocycle of [2] with coefficients in $\mathcal{M} G$. A functor $G:[2] \rightarrow I$ is just a 2 -simplex of the nerve of the category $I$; that is, a system $\left(G i, G_{i, j}: G j \rightarrow G i\right)$, where $G i, i=0,1,2$ are objects of $I$ and $G_{i, j}$, $0 \leqslant i \leqslant j \leqslant 2$, is a morphism in $I$ such that the equation $G_{i, j} G_{j, k}=G_{i, k}$ holds for $0 \leqslant i \leqslant j \leqslant k \leqslant 2$, with $G_{i, i}=1_{G i}, i=0,1,2$. A 2-cocycle of [2] with coefficients in $\mathcal{M} G$ is a system $\left(Y_{i, j}, G_{i, j, k}: G_{j, k}^{*} Y_{i, j} \otimes Y_{j, k} \rightarrow Y_{i, k}\right)$ where $Y_{i, j} \in \mathrm{Ob} \mathcal{M}_{G j}, 0 \leqslant i \leqslant$ $j \leqslant 2$, with $Y_{i, i}=\mathrm{I}$ the unit object of $\mathcal{M}_{G i}$, and where $G_{i, j, k}, 0 \leqslant i \leqslant j \leqslant k \leqslant 2$, are morphisms in $\mathcal{M}_{G k}$ such that, apart from $G_{0,1,2}: G_{1,2}^{*} Y_{0,1} \otimes Y_{1,2} \rightarrow Y_{0,2}, G_{i, i, j}$ and $G_{i, j, j}$, for any $0 \leqslant i<j \leqslant 2$, are given by canonical constraints. All together, we have the following:

$$
\left(\operatorname{Ner}_{I} \mathcal{M}\right)_{2} \cong\left(\Delta^{u} \int_{I} \Omega^{-1} \mathcal{M}\right)_{2}
$$

Finally,

- If $n \geqslant 3$, a $n$-simplex of $\operatorname{Ner}_{I} \mathcal{M}$ is given, on one hand, by a functor $G:[n] \rightarrow I$; that is, a system of data $\left(G i, G_{i, j}: G j \rightarrow G i\right)_{0 \leqslant i<j \leqslant n}$, where $G i \in \mathrm{Ob} I$ and $G_{i, j}$ are morphisms in $I$ such that $G_{i, j} G_{j, k}=G_{i, k}$ for $0 \leqslant i<j<k \leqslant n$ and $G_{i, i}=1_{G i}$ for $0 \leqslant i<j<k \leqslant n$. On the other hand, a 2-cocycle of $[n]$ with coefficients in $\mathcal{M} G$ is given by the system of data $\left(Y_{i, j}, G_{i, j, k}: G_{j, k}^{*} Y_{i, j} \otimes Y_{j, k} \rightarrow Y_{i, k}\right)_{0 \leqslant i<j<k \leqslant n}$, where $Y_{i, j} \in \mathrm{Ob} \mathcal{M}_{G j}$, with $Y_{i, i}=\mathrm{I}$, the unit object of $\mathcal{M}_{G i}$, for each $0 \leqslant i \leqslant n$, and $G_{i, j, k}$ are morphisms in $\mathcal{M}_{G k}$ such that, for any $0 \leqslant i<j \leqslant n, G_{i, i, j}$ and $G_{i, j, j}$ are given by the left and right constraints of the monoidal category $\mathcal{M}_{G j}$ for the object $Y_{i, j}$. Moreover, for all $0 \leqslant i \leqslant j \leqslant k \leqslant \ell \leqslant n$, the coherence condition of (4) must hold, 
that is, the following diagram must be commutative:

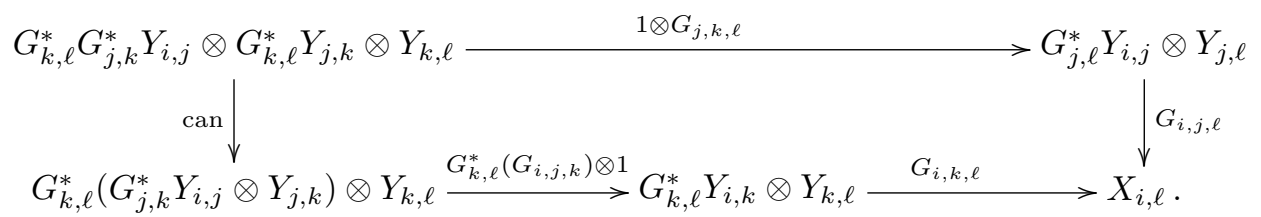

Then, for all $n \geqslant 0$,

$$
\left(\operatorname{Ner}_{I} \mathcal{M}\right)_{n} \cong\left(\Delta^{u} \int_{I} \Omega^{-1} \mathcal{M}\right)_{n}
$$

and so we have the announced isomorphism.

Since the weak homotopy equivalence (3) remains valid taking unitary geometric nerves instead of geometric nerves, the above proposition gives us:

Theorem 2.4. For any diagram of monoidal categories $\mathcal{M}: I^{\mathrm{op}} \rightarrow$ MonCat, there is a natural weak homotopy equivalence

$$
\eta: \operatorname{hocolim}_{I} \Delta^{u} \Omega^{-1} \mathcal{M} \rightarrow \Delta^{u} \int_{I} \Omega^{-1} \mathcal{M} \cong \operatorname{Ner}_{I} \mathcal{M}
$$

where $\Delta^{u} \Omega^{-1} \mathcal{M}: I^{\mathrm{op}} \rightarrow$ Simpl.Set is the diagram of simplicial sets obtained by the composition of $\mathcal{M}$ with the unitary geometric nerve functor $\Delta^{u}$ : Bicat $\rightarrow$ Simpl.Set.

In this way, the geometric realization of $\operatorname{Ner}_{I} \mathcal{M}$ can be thought of as the homotopy colimit of the classifying spaces of the monoidal categories $\mathcal{M}_{i}$ given by the initial data.

\section{Homotopy colimit theorem for braided monoidal categories}

In this section, we give an extension of Theorem 2.4 to braided monoidal categories. Recall that a braided monoidal category, $\mathcal{M}=(\mathcal{M}, \otimes, \boldsymbol{c})$, consists of a monoidal category $\mathcal{M}=(\mathcal{M}, \otimes, a, \mathrm{I}, l, r)$ together with a braiding, that is, a family of natural isomorphisms $\boldsymbol{c}=\boldsymbol{c}_{X, Y}: X \otimes Y \rightarrow Y \otimes X, X, Y \in \mathrm{Ob} \mathcal{M}$, satisfying suitable coherence conditions [14]. The category of braided monoidal categories, and braided monoidal functors between them, is denoted by BrMonCat.

Any braided monoidal category $\mathcal{M}=(\mathcal{M}, \otimes, \boldsymbol{c})$ defines $[\mathbf{3}, \mathbf{1 5}]$, by the categorical delooping principle, a one-object (say $*$ ), one-arrow tricategory $\Omega^{-2} \mathcal{M}$ where the objects of $\mathcal{M}$ are the 2-cells and the morphisms are the 3-cells (thus, $\Omega^{-2} \mathcal{M}(*, *)=$ $\Omega^{-1} \mathcal{M}$ and the braiding provides the interchange 3-cell between the two different composites of 2-cells).

If $\mathcal{B}: I^{\mathrm{op}} \rightarrow$ BrMonCat is a diagram of braided monoidal categories, then the above double delooping construction determines a diagram of tricategories. A hypothetical Grothendieck construction for such a diagram, together with the consideration of suitable nerves, should allow the direct extension of Thomason's Homotopy Colimit Theorem to braided monoidal categories. However, paralleling the monoidal case, we can avoid using the Grothendieck construction to measure the homotopy colimit of the diagram, of simplicial sets, obtained from composing the diagram $\mathcal{B}$ 
with the geometric nerve of braided monoidal categories [6, Definition 6.7]. This is carried out by using the notion of nerve of a pseudofunctor of braided monoidal categories introduced in [7]. In the particular case of considering a diagram $\mathcal{B}: I^{\mathrm{op}} \rightarrow$ BrMonCat, we shall prove that the homotopy type of the diagram can be represented by its nerve, $\operatorname{Ner}_{I} \mathcal{B}$, a simplicial set that we recall below.

A 3-cocycle of $I$ with coefficients in $\mathcal{B}$ is a system of data $(Y, f)$ consisting of:

- For every two composable arrows in $I, k \stackrel{\tau}{\rightarrow} j \stackrel{\sigma}{\rightarrow} i$, an object $Y_{\sigma, \tau}$ of $\mathcal{B}_{k}$.

- For every three composable arrows in $I, \ell \stackrel{\gamma}{\rightarrow} k \stackrel{\tau}{\rightarrow} j \stackrel{\sigma}{\rightarrow} i$, a morphism of $\mathcal{B}_{\ell}$

$$
f_{\sigma, \tau, \gamma}: \gamma^{*} Y_{\sigma, \tau} \otimes Y_{\sigma \tau, \gamma} \rightarrow Y_{\tau, \gamma} \otimes Y_{\sigma, \tau \gamma}
$$

such that, for any four composable arrows in $I, m \stackrel{\delta}{\rightarrow} \ell \stackrel{\gamma}{\rightarrow} k \stackrel{\tau}{\rightarrow} j \stackrel{\sigma}{\rightarrow} i$, the following diagram in $\mathcal{B}_{m}$ (in which we have omitted the associativity constraints) is commutative:

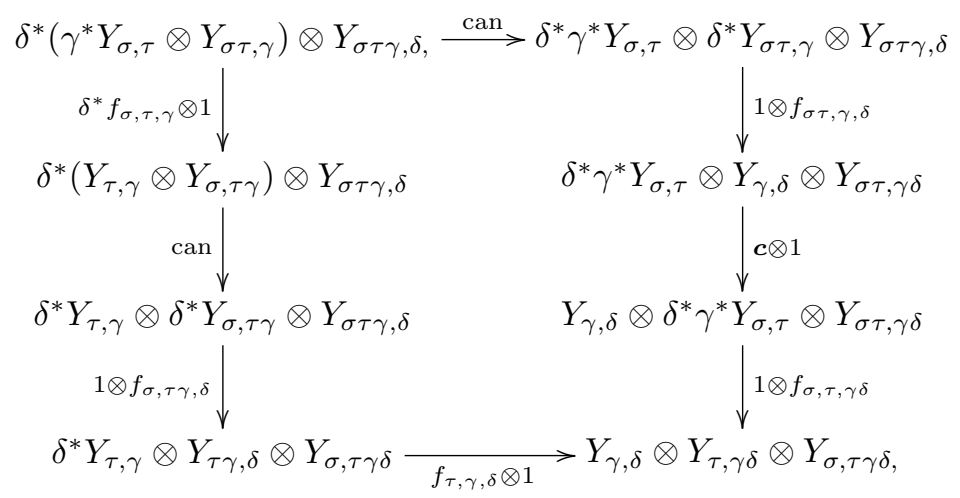

Moreover, $Y_{1, \sigma}=\mathrm{I}=Y_{\sigma, 1}, f_{\sigma, \tau, 1}=c_{Y_{\sigma, \tau}, \mathrm{I}}: Y_{\sigma, \tau} \otimes \mathrm{I} \rightarrow \mathrm{I} \otimes Y_{\sigma, \tau}, f_{\sigma, 1, \gamma}: \gamma^{*} \mathrm{I} \otimes Y_{\sigma, \gamma} \rightarrow$ $\mathrm{I} \otimes Y_{\sigma, \gamma}$ is the composite of $1_{\mathrm{I} \otimes Y_{\sigma, \gamma}}$ with the unit constraint of the monoidal functor $\gamma^{*}$, and $f_{1, \tau, \gamma}: \gamma^{*} \mathrm{I} \otimes Y_{\tau, \gamma} \rightarrow Y_{\tau, \gamma} \otimes \mathrm{I}$ is the composite of $\boldsymbol{c}_{\mathrm{I}, Y_{\tau, \gamma}}$ with the unit constraint of the monoidal functor $\gamma^{*}$.

Then, the nerve $\operatorname{Ner}_{I} \mathcal{B}$ of the diagram is defined as the simplicial set $[\mathbf{7}]$

$$
\operatorname{Ner}_{I} \mathcal{B}:[n] \mapsto \coprod_{G:[n] \rightarrow I} Z^{3}([n], \mathcal{B} G),
$$

where $G:[n] \rightarrow I$ is any functor and $Z^{3}([n], \mathcal{B} G)$ is the set of 3-cocycles of $[n]$ with coefficients in the composite functor $[n] \stackrel{G}{\rightarrow} I \stackrel{\mathcal{B}}{\rightarrow}$ BrMonCat. Thus, an $n$-simplex can be described as a system of data

where

$$
\mathbb{G}=\left\{G_{i, j, k}, G_{i, j, k, \ell}\right\}_{0 \leqslant i \leqslant j \leqslant k \leqslant \ell \leqslant n},
$$

- $G_{i, j, k} \in \mathrm{ObB}_{G k}$ for $0 \leqslant i \leqslant j \leqslant k \leqslant n$, with $G_{i, i, j}=G_{i, j, j}=\mathrm{I}$, the unit object of the braided monoidal category $\mathcal{B}_{G j}$.

- $G_{i, j, k, \ell}: G_{k, \ell}^{*} G_{i, j, k} \otimes G_{i, k, \ell} \rightarrow G_{j, k, \ell} \otimes G_{i, j, \ell}$, for $0 \leqslant i \leqslant j \leqslant k \leqslant \ell \leqslant n$, is a morphism in $\mathcal{B}_{G \ell}$, where $G_{k, \ell}^{*}: \mathcal{B}_{G k} \rightarrow \mathcal{B}_{G \ell}$ is the braided monoidal functor associated to the morphism $\ell \rightarrow k$ of $[n]$, with $G_{i, i, j, k}$ the composite of $\boldsymbol{c}_{\mathrm{I}, G_{i, j, k}}$ with the unit constraint of $G_{j, k}^{*}, G_{i, j, j, k}$ the composite of $1_{\mathrm{I} \otimes G_{i, j, k}}$ with the unit constraint of $G_{j, k}^{*}$ and $G_{i, j, k, k}=\boldsymbol{c}_{G_{i, j, k}, \mathrm{I}}$. 
Moreover, for all $0 \leqslant i \leqslant j \leqslant k \leqslant \ell \leqslant m \leqslant n$, the following diagram in $\mathcal{B}_{G m}$ must be commutative:

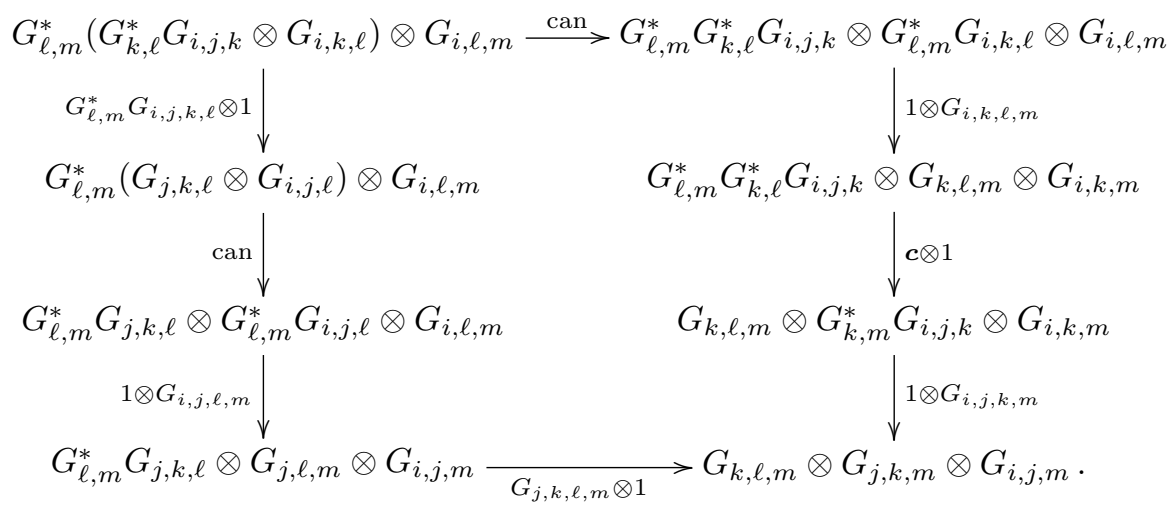

Particularly, if the diagram $\mathcal{B}: I^{\mathrm{op}} \rightarrow$ BrMonCat is a constant braided monoidal category $\mathcal{M}=(\mathcal{M}, \otimes, \boldsymbol{c})$, the notion of a 3-cocycle of $I$ with coefficients in $\mathcal{B}$ is just that of a 3-cocycle of $I$ in $\mathcal{M}$ ([6, Definition 6.6]) and, in this case, $\operatorname{Ner}_{I} \mathcal{B}$ is the geometric nerve, $\operatorname{Ner} \mathcal{M}=Z^{3}(\mathcal{M}, \otimes, \boldsymbol{c})$, of the braided monoidal category $\mathcal{M}$ ([6, Definition 6.7]), that is, the simplicial set

$$
\begin{aligned}
\operatorname{Ner} \mathcal{M}: & \Delta^{\mathrm{op}} \rightarrow \text { Set } \\
& {[n] \mapsto Z^{3}([n], \mathcal{M}), }
\end{aligned}
$$

where $Z^{3}([n], \mathcal{M})$ is the set of 3 -cocycles of $[n]$ in $\mathcal{M}$. This defines the geometric nerve functor of braided monoidal categories, Ner: BrMonCat $\rightarrow$ Simpl.Set, which associates with each braided monoidal category $\mathcal{M}$ its geometric nerve Ner $\mathcal{M}$.

For any diagram $\mathcal{B}: I^{\mathrm{op}} \rightarrow$ BrMonCat, we can consider the bisimplicial set

$$
S=\coprod_{G \in \operatorname{Ner} I} \operatorname{Ner} \mathcal{B}_{G 0}=\coprod_{G:[q] \rightarrow I} Z^{3}\left([p], \mathcal{B}_{G 0}\right)
$$

whose $(p, q)$-simplices are pairs $(\mathbb{G}, G)$, where $G:[q] \rightarrow I$ is a functor and $\mathbb{G}:[p] \rightarrow$ $\mathcal{B}_{G 0}$ is a 3-cocycle of $[p]$ in $\mathcal{B}_{G 0}$. If $\alpha:\left[p^{\prime}\right] \rightarrow[p]$ and $\beta:\left[q^{\prime}\right] \rightarrow[q]$ are maps in the simplicial category, then the respective horizontal and vertical induced maps are defined by

$$
\alpha^{* h}(\mathbb{G}, G)=(\mathbb{G} \alpha, G) ; \alpha^{* v}(\mathbb{G}, G)=\left(G_{0, \beta 0}^{*} \mathbb{G}, G \beta\right),
$$

where $G \beta:\left[q^{\prime}\right] \stackrel{\beta}{\rightarrow}[q] \stackrel{G}{\rightarrow} \mathcal{I}, \mathbb{G} \alpha:\left[p^{\prime}\right] \stackrel{\alpha}{\rightarrow}[p] \stackrel{\mathbb{G}}{\rightarrow} \mathcal{B}_{G 0}$ is a 3-cocycle of $\left[p^{\prime}\right]$ in $\mathcal{B}_{G 0}$ and $G_{0, \beta 0}^{*} \mathbb{G}:[p] \rightarrow \mathcal{B}_{G \beta 0}$ is the 3 -cocycle of $[p]$ in $\mathcal{B}_{G \beta 0}$ obtained by composing $\mathbb{G}$ with the braided monoidal functor $G_{0, \beta 0}^{*}$ associated to the morphism $G \beta 0 \rightarrow G 0$ of $I$. In particular, the horizontal and vertical faces of $S$ are given by

$$
d_{i}^{h}(\mathbb{G}, G)=\left(\mathbb{G} d^{i}, G\right), 0 \leqslant i \leqslant p,
$$

and

$$
d_{j}^{v}(\mathbb{G}, G)=\left(\mathbb{G}, G d^{j}\right), 1 \leqslant j \leqslant q, \quad \text { while } \quad d_{0}^{v}(\mathbb{G}, G)=\left(G_{0,1}^{*} \mathbb{G}, G d^{0}\right) .
$$


The diagonal of this bisimplicial set is just the homotopy colimit, $\operatorname{hocolim}_{I} \operatorname{Ner} \mathcal{B}$, of the diagram of simplicial sets

$$
I^{\text {op }} \stackrel{\mathcal{B}}{\rightarrow} \text { BrMonCat } \stackrel{\text { Ner }}{\longrightarrow} \text { Simpl.Set. }
$$

Thus, $\operatorname{hocolim}_{I} \operatorname{Ner} \mathcal{C}=\operatorname{diag} S$ is the simplicial set whose $n$-simplices are pairs $(\mathbb{G}, G)$, where $G:[n] \rightarrow I$ is a functor and $\mathbb{G}:[n] \rightarrow \mathcal{B}_{G 0}$ is a 3 -cocycle of $[n]$ in $\mathcal{B}_{G 0}$.

Now we are ready to prove our main result:

Theorem 3.1 (Homotopy Colimit Theorem for braided monoidal categories). For any diagram of braided monoidal categories $\mathcal{B}: I^{\mathrm{op}} \rightarrow$ BrMonCat, there exists a natural weak homotopy equivalence of simplicial sets

$$
\eta: \operatorname{hocolim}_{I} \operatorname{Ner} \mathcal{B} \rightarrow \operatorname{Ner}_{I} \mathcal{B},
$$

where Ner $\mathcal{B}: I^{\mathrm{op}} \rightarrow$ Simpl.Set is the diagram of simplicial sets, obtained by the composition of the diagram $\mathcal{B}$ with the geometric nerve functor of braided monoidal categories Ner: BrMonCat $\rightarrow$ Simpl.Set, and $\operatorname{Ner}_{I} \mathcal{B}$ is the nerve (5) of the diagram.

Proof. Let $(\mathbb{G}, G)$ be an $n$-simplex of $\operatorname{hocolim}_{I} \operatorname{Ner} \mathcal{B}$. Then, $G:[n] \rightarrow I$ is a functor and $\mathbb{G}:[n] \rightarrow \mathcal{B}_{G 0}$ is a 3 -cocycle of $[n]$ in $\mathcal{B}_{G 0}$, that is, a system of data

$$
\mathbb{G}=\left\{G_{i, j, k}, G_{i, j, k, \ell}: G_{i, j, k} \otimes G_{i, k, \ell} \rightarrow G_{j, k, \ell} \otimes G_{i, j, \ell}\right\}_{0 \leqslant i \leqslant j \leqslant k \leqslant \ell \leqslant n}
$$

where $G_{i, j, k}$ are objects and $G_{i, j, k, \ell}$ are morphisms of $\mathcal{B}_{G 0}$ such that:

- $G_{i, i, j}=F_{i, j, j}=\mathrm{I}$, the unit object of $\mathcal{B}_{G 0}$;

- $G_{i, i, j, k}$ is the composite of $c_{\mathrm{I}, G_{i, j, k}}$ with the unit constraint of $G_{j, k}^{*}, G_{i, j, j, k}$ is the composite of $1_{\mathrm{I} \otimes G_{i, j, k}}$ with the unit constraint of $G_{j, k}^{*}$ and $G_{i, j, k, k}=\boldsymbol{c}_{G_{i, j, k}, \mathrm{I}}$;

- And, moreover, for all $0 \leqslant i \leqslant j \leqslant k \leqslant \ell \leqslant m \leqslant n$, the following diagram in $\mathcal{B}_{G 0}$ is commutative:

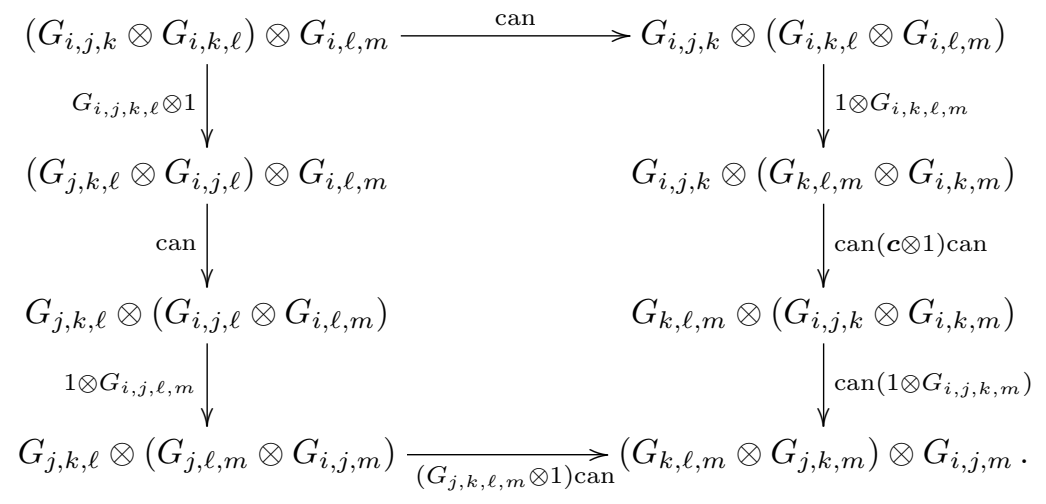

Then we define a map

$$
\eta:\left(\operatorname{hocolim}_{I} \operatorname{Ner} \mathcal{B}\right)_{n} \rightarrow\left(\operatorname{Ner}_{I} \mathcal{B}\right)_{n},(\mathbb{G}, G) \mapsto\left(\mathbb{G}^{\prime}, G\right),
$$

where

$$
\mathbb{G}^{\prime}=\left\{G_{i, j, k}^{\prime}, G_{i, j, k, \ell}^{\prime}: G_{k, \ell}^{*} G_{i, j, k}^{\prime} \otimes G_{i, k, \ell}^{\prime} \rightarrow G_{j, k, \ell}^{\prime} \otimes G_{i, j, \ell}^{\prime}\right\}_{0 \leqslant i \leqslant j \leqslant k \leqslant \ell \leqslant n}
$$

is the 3 -cocycle of $[n]$ with coefficient in $\mathcal{B} G$, defined as follows: 
- The objects of $\mathcal{B}_{G k}, G_{i, j, k}^{\prime}=G_{0, k}^{*} G_{i, j, k}$, for $0 \leqslant i<j<k \leqslant n$, and $G_{i, i, j}^{\prime}=G_{i, j, j}^{\prime}$ $=\mathrm{I}, 0 \leqslant i \leqslant j \leqslant n$, where I denotes the unit object of $\mathcal{B}_{G j}$.

- The morphisms $G_{i, j, k, \ell}^{\prime}$ in $\mathcal{B}_{G \ell}, 0 \leqslant i \leqslant j \leqslant k \leqslant \ell \leqslant n$, given as the dotted arrow in the following diagram:

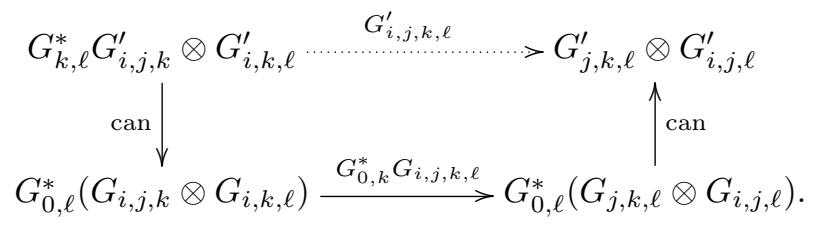

It is straightforward to check that, in this way, $\left(\mathbb{G}^{\prime}, G\right)$ is actually an $n$-simplex of $\operatorname{Ner}_{I} \mathcal{B}$. For instance, the commutativity of diagram (6) for $\mathbb{G}^{\prime}$ is deduced from the commutativity of (7) for $\mathbb{G}$.

Since hocolim $\operatorname{Ner}_{I} \mathcal{B}=\operatorname{diag} S$ we have, according to (1), a natural weak homotopy equivalence $\Phi: \operatorname{hocolim}_{I} \operatorname{Ner} \mathcal{B} \rightarrow \bar{W} S$, and we will prove that $\eta$ is also a weak homotopy equivalence by showing a simplicial isomorphism $\Psi: \bar{W} S \cong \operatorname{Ner}_{I} \mathcal{B}$ making the following diagram of simplicial sets commutative:

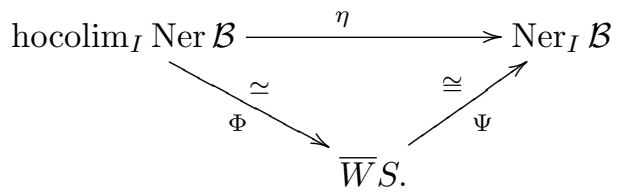

According to the general description of the simplices of $\bar{W} S$ recalled in Section 2, a $p$-simplex of $\bar{W} S$, in our case, can be described as a list of pairs:

$$
\chi=\left(\left(\mathbb{G}^{(0}, G^{(p}\right), \ldots,\left(\mathbb{G}^{(m}, G^{(p-m}\right), \ldots,\left(\mathbb{G}^{(p}, G^{(0}\right)\right)
$$

where each $G^{(p-m}:[p-m] \rightarrow I$ is a functor and each $\mathbb{G}^{(m}:[m] \rightarrow b_{G^{(p-m} 0}$ is a 3-cocycle of $[m]$ in $\mathcal{B}_{G^{(p-m} 0}$, such that the following equalities:

$$
G^{(p-m} d^{0}=G^{(p-m-1}, \quad G_{0,1}^{(p-m *} \mathbb{G}^{(m}=\mathbb{G}^{(m+1} d^{m+1}
$$

hold for all $0 \leqslant m<p$.

Writing $G^{(p}:[p] \rightarrow I$ simply as $G:[p] \rightarrow I$, an iterated use of the above equalities proves that

$$
G^{(p-m}=G d^{0} \stackrel{(m}{\cdots} \cdot d^{0}:[p-m] \rightarrow I, \quad 0 \leqslant m \leqslant p
$$

and

$$
\mathbb{G}^{(m+1} d^{m+1} \cdots d^{k+1}=G_{k, m+1}^{*} \mathbb{G}^{(k}:[k] \rightarrow \mathcal{B}_{G(m+1)} 0 \leqslant k \leqslant m<p .
$$

Since each 3 -cocycle $\mathbb{G}^{(m}$ is a system of data $\left\{G_{i, j, k}^{(m}, G_{i, j, k, \ell}^{(m}\right\}$, these latter equations mean that

$$
G_{i, j, k}^{(\ell}=G_{k, \ell}^{*} G_{i, j, k}^{(k}, i \leqslant j \leqslant k \leqslant \ell ; G_{i, j, k, \ell}^{(m}=G_{\ell, m}^{*} G_{i, j, k, \ell}^{(\ell}, i \leqslant j \leqslant k \leqslant \ell \leqslant m .
$$

Thus, a $p$-simplex $\chi$ of $\bar{W} S$ is uniquely determined by a functor $G:[p] \rightarrow I$, the objects $G_{i, j, k}^{(k)}$ of $\mathcal{B}_{G k}$ and the morphisms $G_{i, j, k, \ell}^{(\ell}$ of $\mathcal{B}_{G \ell}$, for any $0 \leqslant i \leqslant j \leqslant k \leqslant \ell \leqslant p$. Then we observe that there is a 3 -cocycle $\mathbb{G}^{\prime} \stackrel{=}{=}\left\{G_{i, j, k}^{\prime}, G_{i, j, k, \ell}^{\prime}\right\}$ of $[p]$ with coefficients 
in $\mathcal{B} G$ defined by $G_{i, j, k}^{\prime}=G_{i, j, k}^{(k}$, for any $0 \leqslant i<j<k \leqslant p$, and $G_{i, j, k, \ell}^{\prime}=G_{i, j, k, \ell}^{(\ell}$, for any $0 \leqslant i<j<k<\ell \leqslant p$, and therefore a $p$-simplex $\chi$ of $\bar{W} S$ defines a $p$-simplex $\left(\mathbb{G}^{\prime}, G\right)$ of $\operatorname{Ner}_{I} \mathcal{B}$, which itself uniquely determines $\chi$. In this way, we obtain an injective simplicial map $\Psi: \bar{W} S \longrightarrow \operatorname{Ner}_{I} \mathcal{B}$

$$
\left(\left(\mathbb{G}^{(0)}, G^{(p)}\right), \ldots,\left(\mathbb{G}^{(p)}, G^{(0)}\right)\right) \mapsto\left(\mathbb{G}^{\prime}, G\right)=\left(\left\{G_{i, j, k}^{(k)}, G_{i, j, k, l}^{(l)}\right\}, G^{(p)}\right) .
$$

However, $\Psi$ is also surjective. In fact, let $\left(\mathbb{G}^{\prime}, G\right)$ be any $p$-simplex of $\operatorname{Ner}_{I} \mathcal{B}$, that is, let $G:[p] \rightarrow I$ be a functor and let $\mathbb{G}^{\prime}=\left\{G_{i j k}^{\prime}, G_{i j k \ell}^{\prime}\right\}$ be a 3 -cocycle of $[p]$ with coefficients in $\mathcal{B} G$. Then we can consider the $p$-simplex $\chi=\left(\mathbb{G}^{(m}, G^{(p-m}\right)$ of $\bar{W} S$ where, for each $0 \leqslant m \leqslant p, G^{(p-m}:[p-m] \rightarrow I$ is the composite $[p-m] \stackrel{\left(d^{0}\right)^{m}}{\longrightarrow}[p] \stackrel{G}{\longrightarrow} I$ and the 3-cocycle $\mathbb{G}^{(m}$ of $[m]$ in $\mathcal{B}_{G^{\left(p-m_{0}\right.}}$ is defined as follows:

- The objects $G_{i, j, k}^{(m}=G_{k, m}^{*} G_{i, j, k}^{\prime}$;

- The morphisms $G_{i, j, k, \ell}^{(m}: G_{i, j, k}^{(m} \otimes G_{i, k, \ell}^{(m} \rightarrow G_{j, k, \ell}^{(m} \otimes G_{i, j, \ell}^{(m}$ are given as the dotted arrow in the following diagram:

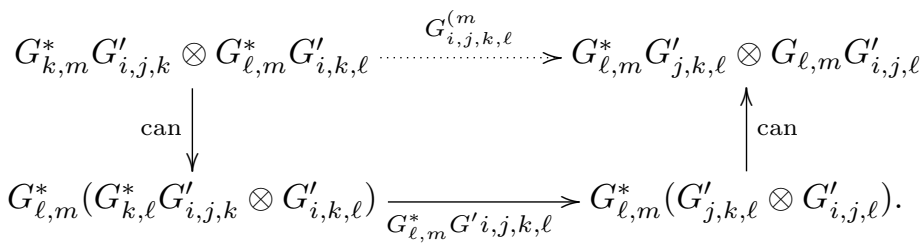

It is easy to check that $\Psi(\chi)=\left(\mathbb{G}^{\prime}, G\right)$, whence we conclude that the simplicial map $\Psi$ is surjective and, therefore it is an isomorphism.

Finally, since $\eta=\Psi \Phi, \Psi$ is an isomorphism and $\Phi$ is a natural weak homotopy equivalence, then $\eta$ is a natural weak homotopy equivalence as claimed.

This theorem allows one to regard the geometric realization of $\operatorname{Ner}_{I} \mathcal{B}$ as the homotopy colimit of the classifying spaces of the braided monoidal categories $\mathcal{B}_{i}$ given by the initial data of the diagram $\mathcal{B}: \mathcal{I}^{\mathrm{op}} \rightarrow$ BrMonCat.

\section{References}

[1] M. Artin and B. Mazur, On the van Kampen theorem, Topology 5 (1966), no. 2, 179-189.

[2] J. Bénabou, Introduction to bicategories, in Reports of the Midwest Category Seminar, pages 1-77, Springer-Verlag, New York, 1967.

[3] C. Berger, Double loop spaces, braided monoidal categories and algebraic 3-type of space, in Higher homotopy structures in topology and mathematical physics (Poughkeepsie, NY, 1996), Contemp. Math. 227, 49-66, Amer. Math. Soc., Providence, RI, 1999.

[4] A.K. Bousfield and D.M. Kan, Homotopy limits, completions and localizations, Lecture Notes in Mathematics 304, Springer-Verlag, New York, 1972.

[5] P. Carrasco, A.M. Cegarra and A.R. Garzón, Nerves and classifying spaces for bicategories, Algeb. Geom. Top. 10 (2010), no. 1, 219-274. 
[6] P. Carrasco, A.M. Cegarra and A.R. Garzón, Classifying spaces for braided monoidal categories and lax diagrams of bicategories, Adv. Math., 226 (2011), no. $1,419-483$.

[7] A.M. Cegarra and E. Khmaladze, Homotopy classification of graded Picard categories, Adv. Math. 213 (2007), no. 2, 644-686.

[8] A.M. Cegarra and J. Remedios, The relationship between the diagonal and the bar constructions on a bisimplicial set, Topology Appl. 153 (2005), no. 1, $21-51$.

[9] A.M. Cegarra and J. Remedios, The behaviour of the $\bar{W}$-construction on the homotopy theory of bisimplicial sets, Manuscripta Math. 124 (2007), no. 4, 427-457.

[10] A.M. Cegarra, Homotopy fiber sequences induced by 2-functors, J. Pure Appl. Algebra 215 (2011), no. 4, 310-334.

[11] P.G. Goerss and J.F. Jardine, Simplicial homotopy theory, Progress in Mathematics 174, Birkhäuser Verlag, Basel, 1999.

[12] R. Gordon, A.J. Power and R. Street, Coherence for tricategories, Mem. Amer. Math. Soc. 117 (1995), no. 558, vi+81pp. Amer. Math. Soc., Providence, RI.

[13] A. Grothendieck, Catégories fibrées et déscente, volume SGA I, Revêtements étales et groupe fondamental, exposé VI, Lecture Notes in Math. 224, 145-194, Springer-Verlag, New York, 1971.

[14] A. Joyal and R. Street, Braided tensor categories, Adv. Math. 102 (1993), no. $1,20-78$.

[15] M.M. Kapranov and V.A. Voevodsky, 2-categories and Zamolodchikov tetrahedra equations, in Algebraic groups and their generalizations: quantum and infinite-dimensional methods (University Park, PA, 1991), Proc. Sympos. Pure Math. 56 (1994), 177-259, Amer. Math. Soc., Providence, RI.

[16] S. Mac Lane, Categories for the working mathematician, second edition, Graduate Texts in Mathematics 5, Springer-Verlag, New York, second 1998.

[17] J.P. May, Simplicial objects in algebraic topology, Van Nostrand Mathematical Studies, No. 11, D. Van Nostrand Co., Inc., Princeton, N.J., 1967.

[18] R. Street, Categorical structures, in Handbook of algebra, Vol. 1, pages 529-577. North-Holland, Amsterdam, 1996.

[19] R.W. Thomason, Homotopy colimits in the category of small categories, Math. Proc. Cambridge Philos. Soc. 85 (1979), no. 1, 91-109.

\section{A.R. Garzón agarzon@ugr.es}

Departamento de Álgebra, Facultad de Ciencias, Universidad de Granada, 18071 Granada, Spain

\section{R. Pérez rocio_pm@ugr.es}

Departamento de Álgebra, Facultad de Ciencias, Universidad de Granada, 18071 Granada, Spain 